\title{
4 Architektur als Protokoll und Protokollierung von Architektur - Methode und Forschungsdesign
}

Die Herausforderungen einer empirischen Architektursoziologie sind nicht nur konstitutionstheoretischer Natur. Architekturanalysen sind auch in methodischer und analytischer Hinsicht eine ambitionierte Aufgabe. Dies liegt zum Ersten daran, dass Architektur synchron und diachron zugleich wirkt. Zum Zweiten liegt es daran, dass Architektur ein nichtsprachliches Protokoll ist. Zum Dritten sind es auch die Größe des Datums, die Art der Protokollierung und Auswertung, die herausfordernde Fragen aufwerfen. Insgesamt verlangt die Architekturanalyse nach experimentellen Forschungsdesigns und Methodenentwicklung (Delitz 2009; Schmidtke 2008).

Die vorliegende Untersuchung verfolgt ein exploratives und qualitativrekonstruktionslogisches Vorgehen, bei dem wenige, ausgewählte Schulanlagen exemplarisch und detailliert analysiert werden. Für beide Problemlagen eignet sich die Methode der Objektiven Hermeneutik (Oevermann 2000a, 2002a; Wagner 2001). Zum Ersten eignet sie sich für die Analyse nichtsprachlicher Protokolle. Zum Zweiten werden in der Rekonstruktion eines spezifischen Falles auch hypothetisch anders gelagerte Fälle jeweils mit expliziert.

Methodisch wird zu weiten Teilen Neuland betreten, denn Sequenzanalysen von Architektur liegen einzig von Schmidtke (2006, 2007) und von Barth (2004a, 2004b) vor. Bei Schmidtke stehen professionalisierungstheoretische Fragen der Ausgestaltung des Arbeitsbündnisses zwischen Architekt und Bauherr im Fokus, was Auswirkungen auf die Art der Protokolle, der Datenpräsentation und auch des sequenziellen Vorgehens hat. Barth verfolgt einen bauhistorischen Zugang, in dem das Werk eines Architekten (Gustav Lüdecke, 18901976) rekonstruiert wird und einer architekturhistorisch-ideengeschichtlichen Verortung gegenübergestellt wird. Die Sequenzanalysen liegen bei Barth jedoch nicht verschriftet vor. Verschriftete und damit intersubjektiv nachvollziehbare Sequenzanalysen eines Luftbilds liegen von Wienke (1999, 2001) vor. Wienke verfolgt explizit eine methodische Frage: Welchen Nutzen kann die Objektive Hermeneutik für siedlungssoziologische Fragestellungen haben? Auf einer konstitutionstheoretischen, methodologischen und methodischen Ebene befasst sich Loer mit den Möglichkeiten der Untersuchung von Siedlungen und Regionen 
(2007), insbesondere der „Eigenlogik einer Stadt“ (2013). Die Überlegungen und Erfahrungen dieser Autoren sind für die Untersuchung bedeutsam und sind daher in die Konzeption des Forschungsdesigns eingeflossen. Da die Analysen nicht direkt am Objekt, sondern anhand von Bildern erfolgen, werden auch methodische Fragen der Sequenzanalyse von Bildern miteinbezogen (Kraimer 2014a; Oevermann 2014; Rittelmeyer \& Wiersing 1991).

In den methodischen Bemerkungen soll es nicht um die Grundlagen der Objektiven Hermeneutik gehen (vgl. dazu Oevermann 1981, 2000a, 2016; Wernet 2009). Ziel der vorliegenden Diskussion ist die Präsentation des methodisch spezifischen Vorgehens, das die sequenzielle Analyse von Architektur betrifft. Im Weiteren werden methodologische und konstitutionstheoretische Fragen dort eingehender diskutiert, wo dies notwendig erscheint oder wo sich methodisch und methodologisch neue Fragen stellen.

Das Kapitel gliedert sich thematisch entlang der methodischen Schritte, wie sie bei der Sequenzanalyse durchgeführt werden: Fallbestimmung, Fallrekonstruktion und Fallerschließung (Kraimer 2014a: 13). Für die methodische Diskussion ist vor allem die Fallerschließung interessant. Die Fallrekonstruktion und die Strukturgeneralisierung werden nachfolgend direkt in den empirischen Fallrekonstruktionen erfolgen.

Die Fallbestimmung klärt formal und inhaltlich, ,was der Fall ist‘. Sie klärt die Fallerfassung und -einbettung mittels geeigneter Protokolle und zugehöriger methodischer Fragen. Der darauffolgende Abschnitt erörtert methodische Fragen der Fallrekonstruktion. Diese betreffen vor allem Fragen der Datenpräsentation und der Sequenzialität in der Analyse. Der letzte Teil behandelt das Forschungsdesign und diskutiert Fragen des Samplings.

\subsection{Was ist der Fall?}

Ziel der rekonstruktionslogischen Analyse ist es, die „Sache zum Sprechen [zu] bringen“ (Wernet 2009: 10). Das heißt, dass die Analyse am konkreten Gegenstand erfolgt. Erst dadurch wird es möglich, das Neue und Fremde - kurz, das Besondere - an einem Fall rekonstruktiv zu entschlüsseln. Das Allgemeine liegt ausschließlich als Besonderes vor. Daher lassen sich auch die allgemeinen Strukturprinzipien erst in der Analyse des Besonderen rekonstruieren. 
Der Fall ist ein zentraler Begriff der Objektiven Hermeneutik; er stellt die Untersuchungseinheit dar. Bevor mit der Sequenzanalyse begonnen wird, ist zu bestimmen, was der interessierende Fall ist, beziehungsweise es wird sich zeigen, dass in der Architekturanalyse fast zwangsläufig mehrere Fälle bedeutsam sind. Formal sind Fälle abzugrenzende Untersuchungseinheiten, die als geschichtlich konstituierte, in sich geschlossene soziale Einheiten erkennbar und deren Grenzen deutlich identifizierbar sind. Der Fall ist im vorliegenden Forschungsprojekt die Architektur von Schulen. Das heißt, Architektur wird als räumlich gebundene Objektivation der Organisation und Institution Schule betrachtet. Dies verlangt nach unterschiedlichen Ebenen der Eingrenzung.

Rein physisch-räumlich gehört die Gesamtheit des architektonisch gestalteten und bebauten Raums eines Perimeters, auf dem die Schulanlage steht, dazu. Dies ist eine physikalische und soziale Ab- beziehungsweise Eingrenzung, die in der Regel durch die Umfriedung markiert ist. Gerade Schulbauten als öffentliche Bauten haben eine Sendung, die weit über den Perimeter hinauswirken. Sie sind zentrale Bauten in dem Siedlungsgefüge, in dem sie stehen. Deshalb wird für die Fallerschließung - und zugleich die Falleinbettung - mit einer Analyse der Umgebung der Schule begonnen, um das Verhältnis der Positionalität der Schule innerhalb des Siedlungsgefüges mitzuberücksichtigen. Für die Analyse der Schule innerhalb des Siedlungsgefüges wird der Kartenausschnitt pragmatisch gewählt, so dass die Fallgesetzlichkeit innerhalb der Siedlung erkennbar ist, aber auch so, dass möglichst viele Details noch erkennbar bleiben.

Die historisch-zeitliche Eingrenzung verfolgt zwei, teilweise konfligierende Merkmale. Die ursprüngliche Konzeption der Arbeit war auch auf eine historische Rekonstruktion der ,Geschichte des Schulhausbaus“ ausgelegt. Die ursprüngliche Idee war, aus den Zyklen der Bildungsexpansionen, die jeweils mit großen Bautätigkeiten einhergingen und -gehen, verschiedene Fallbeispiele zu untersuchen. Dieser Vollständigkeitsanspruch über die historische Genese und die Entwicklungen musste schnell aufgegeben werden und ist im Rahmen dieser Arbeit nicht zu leisten. Es zeigt sich, dass es auch nicht notwendig ist. Denn an einem einzigen Fall sind oft verschiedene Bauetappen manifest vorhanden. Damit wirken auch die Bedeutungsgehalte beziehungsweise die Spurenreste der ursprünglichen Sinnstrukturen in die Gegenwart hinüber. Oder umgekehrt lassen sich auch die ursprünglichen Konzeptionen am Material rekonstruieren. 
Ein weiterer Grund ist die Datenlage in Bezug auf historische Quellen. Für die Zeit der ursprünglichen Erbauung und Nutzung ist die Datenlage teilweise sehr schlecht, lückenhaft oder gar nicht vorhanden. Daher werden die Schulhäuser - mit Ausnahme des Hauses Grün in Kapitel 7.3 - in ihrer heutigen Verwendung betrachtet.

Das Forschungsdesign verfolgt über die Architekturanalyse einen Zugang, dessen Vorannahmen sind, dass sich über die Architektur tief liegende und lang andauernde Sinnstrukturen überliefern. Daher erlauben die zahlreichen umgebauten und neu strukturierten Gebäude und Gebäudeteile einen Rückblick auf das historische Gewordensein, das „vorbewusst bleibt und uns blind beherrscht, wenn es nicht expliziert wird“" (Süßmann 2016: 126).

Als weiterer Fokus soll aus einer methodologischen Perspektive der Fall als Entität eingegrenzt werden. Loer (2013: 63 ff.) unterscheidet drei Möglichkeiten der Fallbestimmung am Untersuchungsgegenstand Stadt, die auch für eine Gebäudegruppe beziehungsweise für eine Schulanlage als verkörperte Sesshaftigkeit einer Schule diskutiert werden sollen. Anzumerken ist, dass Loer mit einem gänzlich anderen Datenmaterial - mit Interviewtranskripten - arbeitet und auch die Fragen der räumlich verorteten Praktiken im Zentrum stehen. Nichtsdestotrotz können an den Ausführungen gewisse Merkmale des Falles herausgearbeitet werden, die für den Gegenstand von Interesse sind. Ich übertrage im Folgenden seine Ausführungen auf den Fall Schule:

Loer unterscheidet drei Fälle: Fall eins ist die Betrachtung einer Schule A als autonome Handlungsinstanz. Die Schule wird als eigenständiger Akteur betrachtet, der eigene handelnde Organe konstituiert. Die Schule stellt sich als Lebenspraxis der Untersuchungseinheit dar, die sich in der Schulanlage als Sesshaftigkeit objektiviert und sich einen passenden Rahmen gibt. Dies entspricht mit gewissen Überlappungen - dem ersten Teil der Fragestellung, welcher die Sinnstrukturen der Sesshaftigkeit und der Positionalität der Schule im Blick hat.

Fall zwei ist die Betrachtung, dass es Praxen und Praktiken einer oder mehrerer Lebenspraxen B in der Schule A gibt, die durch die eigenlogische Struktur der Schule A bestimmt werden. Dieser Fall präsupponiert, dass es schulhausspezifische Praktiken gibt, wie sie beispielsweise das Konzept der Schulkultur beschreibt (Helsper 2015). Dieser Fall ist laut Loer der anzustrebende Fall, auch wenn er methodisch am schwierigsten zu bewerkstelligen ist. Als reine Architekturanalyse ist dieser Fall nur indirekt von Interesse. Die raumabhängigen Prakti- 
ken müssten in geeigneten Protokollen festgehalten und in Bezug zur Sinnstruktur der Schule A gebracht werden. ${ }^{21}$

Fall zwei kann aber indirekt als Bedeutungsraum erschlossen werden, in der Synthese von Fall eins und Fall drei, was für die Architekturanalyse fruchtbar gemacht werden kann.

Fall drei stellt eine Praxis innerhalb einer Schule A dar. Die Schule beziehungsweise das Schulhaus wird als Erzeugungsprinzip eines Möglichkeitsraums von schulhausspezifischen Praktiken und Handlungsoptionen beziehungsweise -dispositionen betrachtet. Architektur emergiert Erzeugungsregeln, die den Möglichkeitsraum von Fall zwei darstellen, ohne dass der konkrete Vollzug dadurch determiniert wird. Architektur hat dabei den Charakter einer Einflussstruktur.

Im sequenzanalytischen Vorgehen ist der Unterschied darin zu sehen, ob die Perspektive auf die realisierten Bedeutungsstrukturen in Form von Architektur im Fall eins gelegt werden oder ob sie auf die daraus emergierenden Sinngehalte für die Akteure in diesem Raum gelegt werden. Oder etwas formalisierter gesagt: Ein Sachverhalt kann unter dem Aspekt betrachtet werden, ob er im Parameter II als Objektivation einer sozialen Repräsentation betrachtet wird oder ob er als Erzeugungsregel im Parameter I für die - in der Sequenzanalyse gedanklich imaginierten - Subjekte im Schulhaus fungiert. In diesem Sinne wird methodisch gesehen die Analyse der Funktionalität der Schulbauten der Analyse der Repräsentation dieser Funktionalität nachgelagert.

Der Fall sind genauer gesagt also immer mehrere Fälle. Es sind die Sinnstrukturen der Architektur einer jeweils in einem Perimeter eingegrenzten Schulanlage und die durch die Architektur erzeugten Bedeutungsstrukturen für die Praktiken der Schule und der Akteure in der Schule.

21 Diese Konzeption verfolgen Böhme und Herrmann (Böhme \& Herrmann 2011; Herrmann \& Flasche 2015), die dementsprechend von einem flüchtigen Interaktionsraum ausgehen, der nur vor Ort wahrnehmbar ist, in dem jedoch die Praktiken abgespeichert vorliegen. Konstitutionstheoretisch kann ich das Konzept nicht nachvollziehen, insbesondere was die Flüchtigkeit des Interaktionsraums angeht. Auch methodisch scheint mir eine Architekturanalyse aufgrund praxeologischer Beobachtungen ein äußerst schwierig zu bewerkstelligendes Unterfangen zu sein. Es scheint mir nur in Ausnahmefällen möglich, Beobachtungen oder Protokollierungen zum Beispiel einer Unterrichtseinheit eindeutig auf architektonische Einflüsse zurückzuführen. Architektur sehe ich eher als Einflussstruktur, die Praktiken mitstrukturiert. Sie ist aber nur Teil vieler möglicher Einflüsse und selten kausale Ursache bestimmter Praktiken. 


\subsection{Zur Protokollierung von Architektur}

Wie bereits mehrfach ausgeführt wurde, ist Architektur ein Artefakt. Artefakte sind methodisch gesehen selbstreferenzielle Protokolle einer Praxis und damit per se authentische Protokolle. Sie sind Produkte und Ausdrucksgestalten der zu untersuchenden Praxis und nicht speziell für die Analyse erzeugte oder edierte Protokolle, wie dies zum Beispiel Interviewtranskripte sind. Artefakte sind daher eine besonders günstige Datenquelle, die als Königsweg der Sozialforschung angesehen werden können. Ein zweites Kriterium für die Güte eines Datenmaterials ist die Frage der Distanz eines Protokolls zur sozialen Alltäglichkeit oder Außeralltäglichkeit. Architektur hat in dieser Hinsicht einen Doppelcharakter. In der Genese ist sie gerade wegen ihrer Dauerhaftigkeit ein sorgfältig ediertes außeralltägliches Protokoll. In der lebenspraktischen Bedeutung kann sie alltäglicher fast nicht sein, da sie in der Regel selten ins Bewusstsein tritt.

Ein weiterer Vorteil von Artefakten ist darin zu sehen, dass sie unter Umständen bereits in Form eines für die Wissenschaft verfügbaren Protokolls vorliegen. Architektur ist ein nichtflüchtiger Gegenstand und damit für eine Analyse verfügbar, die den Prinzipien der Intersubjektivität und damit der Überprüfbarkeit folgt. Das heißt, die Analyse könnte direkt am Gegenstand selbst erfolgen. Dem ist ein großes Aber entgegenzustellen: Die Analyse am Gegenstand wäre sehr umständlich und ginge zulasten der intersubjektiven Nachvollziehbarkeit. Damit diese Nachvollziehbarkeit gewährleistet ist, müssen Protokolle „entzeitlicht“ und „enträumlicht“ werden (Oevermann 2014: 47). Die Schulbauten müssen in eine handhabbare Form gebracht werden, die einer „Doppelung eines Protokolls“ bzw. einer „Protokollierung des Protokolls“ entsprechen (Schmidtke 2008: 4).

Zur Protokollierung von Architektur eignen sich Fotografien und Zeichnungen, insbesondere Pläne des Auf- und Grundrisses. Die Größe und die Schwierigkeiten der rein visuellen Erfassung des Untersuchungsgegenstandes sowie die Mehrdeutigkeit der interessierenden Facetten verlangen nach mehreren Protokollen, die zusammengenommen der Fallrekonstruktion zugrunde gelegt werden. Zudem verlangt auch die Komplexität der Architekturanalyse nach unterschiedlichen Datentypen. Um die Schulanlage als Ganzes zu erfassen, werden Luftbilder, Fotografien und schematische Pläne als Protokolle herbeigezogen. 
Es werden jeweils mehrere Ansichten eines Schulgeländes und -gebäudes zusammen betrachtet. Damit unterscheidet sich die Sequenzanalyse von Architektur methodisch in entscheidenden Punkten von einer Bildanalyse (vgl. dazu Kraimer 2014b). Nicht das einzelne Bild wird analysiert. Das Protokoll bildet die Schulanlage, die mittels einer Serie von Bildern protokolliert wird. Bei Plänen bilden Grund- und Aufrisse das der Sequenzanalyse zugrunde liegende Protokoll.

Da mehrere Bilder das Protokoll bilden, muss die Datenpräsentation, also die vorgängige Auswahl der Bilder diskutiert werden. Zu klären sind dabei die Verfügbarkeit, die Erhebung und Auswahl sowie die Anordnung der Protokolle.

Die Bilder wurden zum größten Teil vom Autor aufgenommen. Zum Teil werden sie ergänzt durch professionelle Fotografien, die von den Architekten oder den Gemeinden zur Verfügung gestellt wurden.

Das zentrale Problem der Sequenzanalyse bei Bildern ist, dass „simultan präsente" Gegenstände (Loer, zit. nach Zollinger 2004: 108), die keine eindeutige Leserichtung innehaben, in Sequenzen zerlegt werden müssen. Denn in der Objektiven Hermeneutik kommt der Einstiegssequenz eine besondere Bedeutung zu. Im nachfolgenden weiteren Material wird die Fallstruktur unter dem Aspekt einer allfälligen Reproduktion oder Transformation untersucht. Bei synchronen Daten ist der Einstieg der Analyse nicht naturwüchsig gegeben, wie dies bei Interaktionen der Fall ist.

Die Problematik entschärft sich, da Architektur eine synchrone und eine diachrone Wirkung hat (vgl. Schmidtke, 2008). Ein Gebäude wirkt als Gesamtheit simultan, und es gibt zugleich sequenzielle Elemente. Zum Ersten sind diese ästhetischer Natur: Es kann unterschieden werden, welche Bauteile oder welche gestalterischen Elemente sich dem Blick des Betrachters aufdrängen. Zum Zweiten sind es die Erschließungswege, über die man sich einem Gebäude annähert und es betritt. Zum Dritten lassen sich in der Architektur gewisse diachrone Elemente in der Baugeschichte festmachen, insbesondere wenn es sich um historisch gewachsene Gebäudegruppen handelt, was bei Schulanlagen gehäuft der Fall ist.

Grundsätzlich kann Architektur von zwei Seiten her interpretiert werden. Von innen her, wobei die funktionalen Aspekte von Architektur in den Fokus rücken. Oder von außen her, wobei die symbolisch-repräsentativen Aspekte im Vordergrund stehen. Beides ist für die Beantwortung der Fragestellung von Inte- 
resse. Und in beiden Fällen muss Gleichzeitiges in Ungleichzeitiges überführt werden. Es gibt keine abschließend einheitliche und eindeutige Antwort, wie vorgegangen werden soll. Schlussendlich muss sich die Methode am Material und am Gegenstand auch bewähren. Entscheidender als die Sequenzfolgen ist, dass die nichttriviale Sequenzialität in der Unterscheidung von eröffneten Möglichkeiten und vollzogener Wirklichkeit strikt eingehalten wird.

Die Sequenzialität wird in dieser Untersuchung von außen nach innen, also von der Siedlungseinbettung zur inneren Raumanordnung festgelegt. Das Vorgehen imitiert eine Annäherung aus der Ferne an das Schulgebäude: Zuerst wird eine Analyse der Siedlungsumgebung und der Morphologie des Schulareals anhand von Luftaufnahmen durchgeführt. Danach werden die Fassaden und Gebäudeerschließungen analysiert. Zuletzt werden die Raumanordnungen und die Binnenbeziehungen innerhalb der Schule betrachtet.

Das Ziel der Analyse der Luftaufnahmen ist, über die Anordnungen der Gebäudekomplexe auf die sozialen Strukturen und Organisationsprinzipien der Siedlung zu schließen. Über die Luftbilder lassen sich auch Aussagen über die historische Genese der Schulen und der Umgebung machen. Die Luftaufnahmen stellen jeweils zwei Ausschnitte unterschiedlichen Maßstabs aus Google Earth dar. Die Auswahl der Ausschnitte wird nach pragmatischen Kriterien getroffen. Es wird ein Ausschnitt gewählt, bei dem die Umgebung ersichtlich und die Details noch erkennbar bleiben. Ein zweites Bild fokussiert jeweils das Schulgelände, um die Detailtiefe zu vergrößern. Die Luftbilder sind hochgradig edierte Protokolle, die aus unzähligen zusammengesetzten Fotos und aus technischautomatisch durchgeführten Retuschen bestehen. Dabei ist anzufügen, dass sich Google Earth während der Zeit der Untersuchung stark verändert hat, sowohl was die Auflösung und damit die Detaildichte betrifft als aber auch bezüglich der technischen Betrachtungsmöglichkeiten. Um ein entzeitlichtes und enträumlichtes Protokoll zu erhalten, war es daher notwendig, die Protokolle in Form von Bildschirmfotografien festzuhalten.

Der zweite Schritt der Sequenzanalyse stellt die Analyse der äußerlichen Gestalt der Schulen anhand von Fotografien dar. Da bei der Analyse von Bildern die Sequenzialität nicht wie bei der Schrift in einer natürlichen Abfolge gegeben ist, wird sie innerhalb der einzelnen Bilder nach methodischen Kriterien festgelegt. Die Abfolge der Fotografien soll einer möglichst naturwüchsigen Annäherung entsprechen: Fassaden, Eingangsbereiche, Innenräume. Das entspricht dem 
Betreten des Geländes und der Gebäude. Als weiteres Element der Datenpräsentation werden bei diesem Analyseschritt die Fotografien der gegenüberliegenden Seiten ergänzend/kontrastierend gegenübergestellt. Dies ist notwendig, da die Symmetrie/Asymmetrie der Gebäude zentral erscheint. Zudem können so die Schulanlagen oder die Gebäude als Ganzes erfasst werden.

Der dritte Schritt der Rekonstruktion der Schulbauarchitektur ist die Analyse der Binnenbeziehungen über die Ortsrelationen und Grundrisse. Dazu eignen sich Pläne. Karten und Pläne sind Zeichnungen, die einen bestimmten Zweck haben. Wie Oevermann (2014: 57) am Beispiel von Landkarten ausführt, sind Karten ,nicht nur Protokolle der Erdoberfläche, sondern vor allem Protokolle eines kodifizierten Wissens darüber“. Pläne können als Blaupausen - als Urmodelle - einer ideal-hypothetischen Praxis gelesen werden. Die Analyse der Grundrisse ist insofern zentral, als sie eine implizite, aber auch eine explizite ,Theorie der Schule "über die Funktionsräume beziehungsweise über die Funktionsbestimmungen der Räume enthält. Die Funktionen der Räume werden als Programmatik einer Schule gelesen werden, während die Raumanordnungen viel über die Binnenbeziehungen der Akteure und die schulische Ordnungsstruktur aussagen.

Bereits festgehalten wurde, dass die Analyse von Architektur nach einer Analyse unterschiedlicher Datentypen verlangt. Die Gemeinsamkeit der Datentypen ist, dass sie allesamt nichtsprachliche Ausdrucksgestalten sind. Vor der eigentlichen Analyse nichtsprachlicher Ausdruckgestalten ist eine Beschreibung des Dargestellten unumgänglich. Bei Bildern kann das Abgebildete zwar sinnlich wahrgenommen werden, für eine intersubjektiv nachvollziehbare Analyse braucht es aber eine sprachliche Erläuterung einer Objektbeschreibung, welche die Bauhistorie, Bezüge zum Außen, die Positionalität, die formalen Eigenschaften wie das Raumprogramm, die Grundrissgestaltung und die Fassadenabwicklung beinhaltet. Oder wie Barth (2004a: 57) es festhält: Vor der Analyse muss in Sprache gefasst werden, ,was faktisch dargestellt, normativ beinhaltet und expressiv ausgedrückt wird“. Damit wird zugleich das Sehen vom Wissen kategorial getrennt. Die Versprachlichung und Vermittlung der Architektur über Texte, Bilder oder Filme ist bei der Architektur aufgrund der Ortsgebundenheit und der Exklusivität ihrer Zugänglichkeiten ohnehin einer der häufigste Weg ihrer Erfahrung. 


\subsection{Sequenzanalyse nichtsprachlicher, simultan präsenter Protokolle}

Sequenzanalyse besteht im sequenziellen Vorgehen. Die sequenzielle Analyse von synchronen Daten ist insofern eine Krux, als das Datenmaterial in Sequenzen eingeteilt werden muss, die einer nichttrivialen Sequenzialität entsprechen. Denn wesentlich an der Sequenzanalyse ist nicht die Sequenzialität des zeitlichen Nacheinander, sondern die analytische Trennung von Parameter I und Parameter II, also die Wahl aus mehreren durch Regeln erzeugten alternativen Möglichkeiten. Für das Vorgehen ist nicht der Nachvollzug einer trivialen Sequenzialität in der Aneinanderreihung wesentlich, sondern die Sichtbarmachung der vorhandenen Möglichkeiten innerhalb der bedeutungsgenerierenden Eröffnungsprozeduren und den vollzogenen Beschließungen. Und zum Zweiten sind die Bedeutungsstrukturen der realisierten Praxis - also der getroffenen Auswahl -, aufgrund der erzeugenden Regeln zu interpretieren. Der Sequenzanalyse liegt das in Kapitel 2.1 beschriebene Krisenmodell zugrunde. Bereits mehrfach erwähnt wurde, dass lebenspraktisch die Routine den Normalfall und die Krise den Ausnahmefall darstellt, konstitutionstheoretisch und methodisch wird dieser Umstand umgedreht.

„Sozialwissenschaftlich theoretische Erklärungen liegen meines Ermessens erst vor, wenn ihre Begriffe und Methoden es gestatten, den Gegenstand, die menschliche Lebenspraxis, so weit verfremdend - auf analytische Distanz zu bringen, dass im Gegensatz zur Perspektive der Lebenspraxis selbst, die Krise als der Normalfall erscheint und die Routine als der davon abgeleitete Grenzfall. Routinen werden nicht als Routinen geboren, sondern ergeben sich aus ursprünglichen Krisenlösungen unter der Bedingung der Bewährung und Veralltäglichung." (Oevermann 2000b: 447)

Es geht bei der Analyse laut Oevermann im Wesentlichen darum, an jeder Sequenzstelle, an der Entscheidungen getroffen wurden, nicht nur die vollzogene Entscheidung, sondern auch die durch Regeln entstehenden alternativen Möglichkeiten zu rekonstruieren. Am Beispiel eines Anbaus oder der Platzierung einer Tür wird gedankenexperimentell expliziert, welche Möglichkeiten objektiv vorhanden sind und welche Bedeutungsstrukturen die verschiedenen Möglichkeiten darstellen. In der Ausformulierung der verschiedenen Möglichkeiten wird die Bedeutungsstruktur der vollzogenen Realisierung als Teil der eigengesetzlichen Fallstruktur fassbar. Dabei eröffnet die eine realisierte Krisenlösung, z. B. 
die Platzierung der Tür, erneut verschiedene Möglichkeiten der Erschließungswege, der Raumanordnungen.

Die Rekonstruktion und Ausbuchstabierung der objektiv gegebenen Möglichkeiten an jeder Sequenzstelle hat einerseits zur Folge, dass nicht nur der betrachtete Fall, sondern auch die hypothetisch möglichen Fälle mitexpliziert werden. Andererseits tritt die Sinnstruktur der gewählten Realisation im Kontrast zu den gegebenen Möglichkeiten erst im Kontext der vorhandenen Alternativen geschärft in den Fokus. Also: Die Bedeutung der Platzierung eines Gebäudes innerhalb des verfügbaren Perimeters lässt sich erst dann erschließen, wenn die objektiv gegebenen Möglichkeiten mitbetrachtet werden und deren Bedeutung mitrekonstruiert wird.

An jeder Sequenzstelle werden nun also Lesarten gebildet, die den objektiv gegebenen, wohlgeformten Möglichkeiten entsprechen. Vor dieser Grundlage werden die Bedeutungsgehalte erkennbar, die mit der vollzogenen Möglichkeit realisiert wurden. Dies ist insofern zentral, als ,die Bedeutung (...) sich also nicht einfach abgelöst von der Sequenzstelle als eine generalisierte Festlegung [ergibt] (...), sondern sie stellt eine Verbindung von generalisierter Explikation und Bestimmung der Einbettung in eine Sequenzfolge dar" (2008: 3). Erst mit der Explikation der möglichen Fälle und der sequenziellen Analyse der sich reproduzierenden Sinnstrukturen wird die Bedeutungsstruktur des konkreten Falles erschlossen.

Innerhalb der Protokolle sind unterschiedliche Vorgehensweisen in der Sequenzanalyse denkbar. Bei Gemälden, Bildern und Fotografien kann zuerst das ikonische Zentrum analysiert werden, um danach dem ikonischen Pfad des Bildes zu folgen (Fehlhaber \& Kirsch 2014: 165). Haupert (1994) hingegen schlägt vor, bei der Analyse von Fotografien zuerst den Gesamteindruck zu schildern, um danach ähnlich wie beim Schriftbild von oben links nach unten rechts das Bild abzuarbeiten. Schmidtke folgt der Sequenzialität, die sich „durch Hierarchien der Aufmerksamkeit“" (Schmidtke 2008: 2) strukturiert. Bei den vorliegenden Analysen wurde eine Mischform praktiziert. Bei den Luftaufnahmen werden die Schulgebäude bewusst ins ,ikonische Zentrum' gerückt. Auch bei den Fotografien steht ein bewusst gewähltes Zentrum im Fokus der Aufnahme. Für das sequenzielle Vorgehen wurde je nach Datentyp ein unterschiedlicher Zugang gewählt. Bei Luftbildern wird zuerst die Umgebung, also die Einbettung der Schule analysiert, da vor allem der soziale Kontext einer Schule rekonstruiert 
werden soll. Bei der Fokussierung auf das Schulgelände wird die Sequenzialität entlang der visuellen Mächtigkeit vorgenommen: Das, was zuerst ins Auge sticht, wird auch zuerst analysiert. Früher oder später wird in jeder Analyse die Frage der Chronologie in der Genese der Schulanlagen aufgeworfen, zumindest dort, wo An- und Zusatzbauten aus unterschiedlichen Zeiten errichtet wurden. Somit wird auch die historisch gegebene Abfolge der Baukörper für die Sequenzanalyse prägend sein.

Bei den Fotografien und den Plänen wird die Sequenzialität auch aufgrund der visuell auffälligen und herausragenden Elemente festgelegt. Grundsätzlich kann aber die rein formale Festlegung des sequenziellen Vorgehens vernachlässigt werden, wenn die Prinzipien der Sequenzanalyse streng eingehalten werden. Die Abfolge ergibt sich im Vorgehen meist naturwüchsig, da die Sequenzanalyse die Sequenzialität nicht als triviale Abfolge von Elementen betrachtet, sondern die Verbundenheit dieser Elemente innerhalb eines Verweisungszusammenhangs in den Blick rückt (siehe auch Schmidtke 2008).

Für die Analyse der Binnenfunktionen wird auch auf Pläne zurückgegriffen. Im Unterschied zu Fotografien sind Pläne ein Datum, bei dem nicht unterschieden werden kann, ob es Abbild einer Realisation ist oder nicht. Pläne sind Entwürfe, das heißt Imaginationen. Zugleich sind es Idealvorstellungen, die unter Umständen, aus welchen Gründen auch immer, nicht so realisiert werden, wie sie geplant werden. In diesem Sinne sind Pläne als Datenmaterial im Gegensatz zu Fotografien oder zum empirischen Objekt mit einem Informationsdefizit belastet. Zugleich ist der entscheidende Vorteil von Plänen, dass sie einen beachtlichen Informationsgewinn geben, indem sie eine An- und Übersicht über ein Objekt erlauben, welche empirisch nicht realisiert werden kann. Ein Grundriss mit Raumprogramm ist empirisch nie visuell erfassbar. Pläne machen Unsichtbares nicht sichtbar, aber vorstellbar und damit greifbar.

\subsection{Die Auswahl der Fälle}

Die Auswahl der Fälle wurde nach dem Vorgehen des theoretical sampling (Corbin \& Strauss 2008) und der maximalen Kontrastierung durchgeführt. Das heißt, es wurde jeweils ein Fall erhoben und analysiert und danach aufgrund der Ergebnisse ein weiteres Schulhaus mit dem größtmöglichen Kontrast gesucht. 
Das Schweizer Bildungswesen ist horizontal und vertikal stark stratifiziert. Das Forschungsvorhaben konzentriert sich auf Schulanlagen der Primarschule. Die Primarschule entspricht in ihrer heutigen Form etwa der deutschen Grundschule. Die Hoheit über die Schule liegt in der Schweiz bei den 26 Kantonen. Die Harmonisierungsbestrebungen seit 2006 sehen eine einheitliche Stufeneinteilung vor, wobei sie zehn Jahre später noch nicht überall restlos umgesetzt ist. In einigen Schweizer Kantonen gibt oder gab es bis dahin ein dreistufiges System. Ursprünglich sollte die Untersuchung auf die Grundstufe (1.- 4. Klasse) beschränkt werden. Dies hat sich als nicht praktikabel erwiesen, insbesondere da Schulbauten nur für die Unterstufe (noch) die Ausnahme sind. Die Idee war, dass sich in der Architektur für Schulbauten der Unterstufe Deutungsmuster zur Sozialisation durch Schule besonders konturiert zeigen müssten, weil jüngere Kinder beim Schuleintritt aus Altersgründen und des damit verbundenen Entwicklungsstandes am ehesten nach einer schulspezifisch eigenen Architektur verlangen. Dies legt zumindest eine heutige Perspektive nahe, die vom Diskurs einer kindzentrierten und kindgerechten Schule dominiert wird, obwohl keineswegs einheitlich ist, was faktisch genau darunter verstanden wird.

Relativ schnell hat sich bereits bei der Auswahl der Fälle gezeigt, dass schon die Einengung auf Primarschulen keinesfalls heißt, dass die betreffenden Gebäude auch als Primarschulen erbaut wurden. Im Gegenteil. Viele Primarschulhäuser sind ursprünglich für die Sekundarschule erbaut worden und wurden später einer Umwidmung zugeführt.

Diese Feststellungen machten für das methodische Vorgehen einige entscheidende Weichenstellungen nötig. Allen voran ist es die Frage, ob die Schulbauten gegenwartsdiagnostisch in ihrer heutigen Nutzung protokolliert und analysiert oder ob sie in ihrer historischen Entstehung und anhand historischen Datenmaterials analysiert werden sollen. Faktisch habe ich mich auch hier für eine Mischform entschieden. Die meisten Schulhäuser werden in ihrem gegenwärtigen Zustand analysiert. Dies ergibt sich einerseits aus den Fragestellungen beziehungsweise aus der Konzeption des Forschungsdesigns über den architektursoziologischen Zugang. Es wird ja gerade explizit die These vertreten und untersucht, dass Architektur als Speicher und dauerhafte Routine für die Konstanz der Bedeutungsstrukturen in der Schule prägend ist. Zudem hatten auch die ersten Analysen gezeigt, dass sich die historisch-kulturell verankerten Umbrüche in den Schulbauten objektivieren und somit rekonstruiert werden können. Das histori- 
sche Quellenmaterial wird aber - sofern es überhaupt vorhanden ist - zur Klärung von strittigen Punkten und bei der historischen Rekonstruktion der Ergebnisse in die Analysen einbezogen. Die historischen Daten zu den einzelnen Schulhäusern sind zum Teil lückenhaft oder nicht zugänglich. Für die beiden ältesten Schulhäuser in Schmitten wurde eine Rekonstruktion in der historischen Genese durchgeführt, auch wenn das Datenmaterial sehr lückenhaft ist. Es ergibt insofern Sinn, als die Häuser heute nicht mehr als Schulgebäude genutzt werden. Als Teil des Ensembles der Schulanlage Schmitten und als Datenmaterial über die Genese der öffentlich-rechtlichen Schule sind sie aber von großem Interesse und bringen zusätzliche Erkenntnisse.

$\mathrm{Zu}$ Beginn der Untersuchung wurden verschiedene Schulhäuser und Teile von Schulanlagen einer Sequenzanalyse unterzogen, um die Methode zu erproben. In diesen Fallrekonstruktionen haben mich vor allem die Strukturprinzipien des Gebäudetyps Schulhaus interessiert. Aus diesen Analysen wurde das Schulhaus Breitenrain in der Stadt Bern als erster Fall komplett verschriftet. Das Schulhaus Breitenrain wurde ausgewählt, da es als relativ unspektakuläres Schulhaus, als prototypische Schulkaserne gelten kann, die für die Erbauungszeit typisch ist und die auch heute noch zahlreich in Gebrauch ist. ${ }^{22}$ Zudem machen die zahlreichen An- und Umbauten das Breitenrain-Schulhaus interessant, da sie die Strukturtransformationen abbilden, die das Schulhaus in seiner heutigen Nutzung prägen. Als dritter Punkt interessierte mich die relative Platzknappheit, die dadurch gelöst wurde, dass das Schulhausareal in den nebenan liegenden Park ausgedehnt wurde.

Danach wurde ein maximal kontrastierender Fall gesucht. Das Kriterium war, ein möglichst neues Schulhaus zu finden, das den Zeitgeist, der im Diskurs geforderten ,neuen, modernen Schule' abbilden sollte. Damit werden insbesondere Architekturen bezeichnet, die für kooperative und kollaborative Lernformen entwickelt werden, die flexiblen Raumkonzepten entsprechen sollen, die als Lern- oder Bildungslandschaften benannt werden (z. B. bei Braun et al. 2014; Montag Stiftung Jugend und Gesellschaft Bonn 2011). Dabei bin ich etwa zeit-

22 Für die nicht schweizerischen Leser_innen ist hervorzuheben, dass die Schweiz von Kriegsschäden unbelastet ist. So sind viele Gebäude des öffentlichen Schulwesens intakt und in Gebrauch. Neu- und Erweiterungsbauten sind in erster Linie auf demographische Entwicklungen oder auf Bildungsexpansionen zurückzuführen. 
gleich auf die Schulhäuser Hinter Gärten in Riehen (Kanton Basel-Stadt) und auf das Schulhaus Gelb in Schmitten (Kanton Freiburg) gestoßen. Das Schulhaus Hinter Gärten wurde ausgewählt, da es als eine Neukonzeption an einem neuen Standort errichtet wurde. Es gibt also keinen Bestand, wie die Architekten sagen, der in der Planung berücksichtigt werden müsste.

An den Schulhäusern Schmitten ist mir insbesondere aufgefallen, dass rund zehn Gebäude vorhanden sind, die historisch gesehen das öffentlich-rechtliche Schulwesen nahtlos abdecken. Es kann an diesem einen Fall weitestgehend die Genese und der Wandel von Schulbauten nachvollzogen und entziffert werden. Zudem können die Analysen auch mit der analysierten Siedlungsgenese verbunden werden, da die Gemeinde Schmitten eine überschaubare Größe aufweist. Am Fall Schmitten können sowohl zeitdiagnostische als auch historische Komponente des Forschungsvorhabens rekonstruiert werden. Auch ergibt sich mit dem ländlichen Schmitten eine kontrastive Ergänzung zu den städtischen Schulbauten und denjenigen aus der Agglomeration.

Da das Forschungsvorhaben explizit auch ein methodisches Unterfangen ist, ist es mir wichtig, die Fälle analysezentriert darzustellen. Das hat zur Folge, dass nicht alle Fälle verschriftet werden können und dass die verschrifteten Fälle viel Platz einnehmen. Aus Platzgründen kann beim Fall Schmitten lediglich eine Auswahl der Schulhäuser analysezentriert dargestellt werden.

\subsection{Zur Verschriftlichung und Darstellung der Fallanalysen}

Die Fälle werden analysezentriert dargestellt. Diese Darstellung verlangt der Leserin und dem Leser einiges ab. Der Vorteil einer sequenzanalytischen Darstellung ist, dass die Intersubjektivität größtmöglich gewährleistet wird und dass direkt am Datenmaterial dessen Eigenlogik rekonstruiert werden kann. Nachteilig wirkt sich aus, dass Wiederholungen unumgänglich sind, und zwar nicht nur dort, wo sie sich am Material reproduzieren. Häufig wird es auch unumgänglich sein, auf bestimmte Aspekte mehrmals zurückzugreifen. Eine Herausforderung ist die schiere Größe und Komplexität des Gegenstandes. Architektur wird auch alltagspraktisch nicht als Gesamtes erfasst, sondern gerät immer als (Bruch-)Teil des Ganzen, z. B. als Fassade, als Korridor, als Garderobe usw., in den Blick. Sie kann auch visuell nicht aus einer Perspektive erfasst werden, sondern nur über 
unterschiedlichste Perspektiven, Ansichten und sinnliche Eindrücke. Sie muss in kleine Teilbereiche zerlegt werden, um sie überhaupt als Datenmaterial verwenden zu können. Erschwerend kommt hinzu, dass Architektur eine nichtsprachliche Ausdrucksgestalt ist. Sie wirkt synchron und muss in der Versprachlichung in diachrone Teilstücke zerlegt werden. Aus Gründen der Synchronizität des Gegenstandes und der Daten müssen zahlreiche Kompromisse gemacht werden, um überhaupt einen lesbaren Text zustande zu bekommen. Die Texterstellung ist ein ständiges Abwägen zwischen extensiver Auslegung und pragmatischer $\mathrm{Zu}$ spitzung und Verkürzung, zwischen ausschweifenden Rekonstruktionen und abkürzenden Illustrationen.

Die Darstellungsstruktur der Fälle folgt einer sukzessiven Annäherung an die Gebäude. Diese wird in unterschiedliche Segmente zerlegt, was auch in unterschiedlichen Textsegmenten resultiert. Ein erster Teil ist der Fokus auf die Siedlungsstruktur, die um die Schulanlagen zentriert sind. Als zweites Segment folgt eine Konzentration auf das Schulgelände. Als drittes Segment wird der Fokus auf die Schulgebäude und deren Repräsentation nach außen gelegt. Als viertes und letztes Segment folgt die Betrachtung der Binnenfunktion des Gebäudes in Form von Raumprogramm und -anordnungen. Dies ist die idealtypische Aufteilung. Im Text kann dies nicht überall strikt durchgehalten werden. Teilweise sind Vor- oder Rückgriffe notwendig, um gewisse Ergebnisse konturierter darzustellen. Insbesondere beim Fall Schmitten konzentriert sich die Verschriftung auf diejenigen Elemente, die Neues hervorbringen.

Jedes Segment ist weiter unterteilt. Den Fällen wird ihre Bauhistorie vorangestellt. Sie erlauben eine Situierung des Falles in seinen Rahmenbedingungen. In den Paraphrasen wird sprachlich ausgedrückt, was in den Protokollen dargestellt ist, was diese Darstellungen normativ beinhalten und was sie expressiv ausdrücken. Diese Darstellungslogik wird von Barth (2004a) übernommen. Die Paraphrasen werden möglichst neutral gehalten. Das Ziel ist die - notwendigerweise selektive und unvollständige - Versprachlichung des Datenmaterials. Die Paraphrasen werden möglichst frei von Interpretationen den Analysen vorangestellt. Die Analysen sind wo notwendig durch Einschübe, Exkurse und Zwischenergebnisse unterbrochen.

Für den intersubjektiven Nachvollzug der Architekturanalysen sind Bilder unverzichtbar, und manchmal einfach unterstützend. 
Wie Barth es ausdrückt, steht ,,am Ende des theoretischen Horizonts (...) immer die Erkenntnis, dass sich gesellschaftlich-historische Vorgänge nur im Rahmen praktischer Absichten erschließen lassen“ (Barth 2004b: 2). Im Weiteren werden die praktischen Absichten anhand der materialisierten Schulbauten erschlossen. In diesem Sinne fällt hier der Vorhang für die empirischen Analysen.

Open Access Dieses Kapitel wird unter der Creative Commons Namensnennung 4.0 International Lizenz (http://creativecommons.org/licenses/by/4.0/deed.de) veröffentlicht, welche die Nutzung, Vervielfältigung, Bearbeitung, Verbreitung und Wiedergabe in jeglichem Medium und Format erlaubt, sofern Sie den/die ursprünglichen Autor(en) und die Quelle ordnungsgemäß nennen, einen Link zur Creative Commons Lizenz beifügen und angeben, ob Änderungen vorgenommen wurden.

Die in diesem Kapitel enthaltenen Bilder und sonstiges Drittmaterial unterliegen ebenfalls der genannten Creative Commons Lizenz, sofern sich aus der Abbildungslegende nichts anderes ergibt. Sofern das betreffende Material nicht unter der genannten Creative Commons Lizenz steht und die betreffende Handlung nicht nach gesetzlichen Vorschriften erlaubt ist, ist für die oben aufgeführten Weiterverwendungen des Materials die Einwilligung des jeweiligen Rechteinhabers einzuholen.

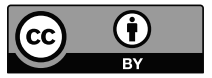

